\title{
Primary Rectal Mucosa-Associated Lymphoid Tissue Lymphoma: Report of a Case with Extensive FISH Study
}

\author{
Wei-Shou Hwang ${ }^{1}$, Hongtao $\mathrm{Ye}^{2}$, Shu-Hui Lin ${ }^{3}$ and Shih-Sung Chuang ${ }^{*}, 3,4$ \\ ${ }^{I}$ Division of Hemato-oncology, Department of Internal Medicine, Chi-Mei Medical Center, Tainan, Taiwan \\ ${ }^{2}$ Department of Pathology, University of Cambridge, Cambridge, United Kingdom. \\ ${ }^{3}$ Department of Pathology, Chi-Mei Medical Center, Tainan, Taiwan \\ ${ }^{3}$ Department of Pathology, Taipei Medical University, Taipei, Taiwan
}

\begin{abstract}
Primary rectal mucosa-associated lymphoid tissue (MALT) lymphoma is a very rare entity with scarce cytogenetic/molecular genetic data. We presented a polypoid rectal MALT lymphoma in a 75 year-old male. The tumor comprised small atypical lymphocytes forming focal lymphoepithelial lesions and expressing CD20 but not CD5, CD23, CD43, or cyclin D1. They were negative for translocations involving IGH, MALT1, BCL2, BCL6, and CCND1 genes, and the copy numbers of chromosomes 3,12 , and 18 were normal by fluorescent in situ hybridization. The patient was disease-free for 83 months after oral endoxan and prednisolone and concurrent local radiotherapy. With increasing awareness of this disease and accumulating reports on clinicopathological and molecular data, the pathogenesis, best treatment strategy and clinical course of such tumors will be uncovered.
\end{abstract}

Keywords: Fluorescence in situ hybridization, mucosa-associated lymphoid tissue lymphoma, non-Hodgkin lymphoma, rectum, Taiwan.

\section{INTRODUCTION}

Mucosa-associated lymphoid tissue (MALT) lymphoma is low-grade B-cell lymphomas with histological characteristics recapitulating those of MALT as exemplified by the Peyer's patches [1]. Excluding diffuse large B-cell lymphoma, most intestinal B-cell lymphomas are of MALT type with the majority arising in the small intestine and rarely in the colorectal region $[2,3]$. Primary rectal MALT lymphomas account for less than $1 \%$ of MALT lymphomas with only 30 more cases been reported in the English literature [4, 5]. The majority was polypoid lesions with a favorable prognosis; however, cytogenetic/molecular genetic study has rarely been performed. So far the only chromosomal aberration detected in rectal MALT lymphoma is $\mathrm{t}(11 ; 18)$, in only $11 \%$ of cases [6-8]. We presented a case of rectal MALT lymphoma with extensive immunohistochemical and fluorescent in situ hybridization (FISH) studies and long-term follow-up information.

\section{REPORT OF A CASE}

A 75 year-old male presented with bowel habit change for 7-8 months without body weight loss, fever, or night sweating. He had no history of inflammatory bowel disease or any major illness. Digital examination revealed a palpable tumor, $5 \mathrm{~cm}$ from anal verge. Physical examination was negative for lymphadenopathy. During operation, a widebased, 3-cm polypoid tumor with a cauliflower appearance and easy touch bleeding was identified and polypectomy was

\footnotetext{
*Address correspondence to this author at the Department of Pathology, Chi-Mei Medical Center, 901 Chung-Hwa Road, Yung-Kang City, Tainan County, Taiwan 710; Tel: 886-6-281-2811, Ext. 53686; Fax: 886-6-2511235 ; E-mail: cmh5301@mail.chimei.org.tw
}

performed. A diagnosis of malignant lymphoma was made and staging procedures showed an IE disease. The LDH level was within normal ranges. The patient received oral cyclophosphamide and prednisolone and concurrent local radiotherapy for $4320 \mathrm{cGy}$ in 15 fractions. He was free of lymphoma for 83 months.

Under low-power microscopic examination, the polypectomy specimen showed vaguely nodular lymphoid infiltrate markedly expanding the submucosa with extension into the muscularis propria (Fig. 1A). The surface mucosa was intact without ulceration. Under high-power, occasional lymphoepithelial lesions were seen (Fig. 1B). The medium-sized lymphocytes exhibited irregular nuclear contours and moderate amount of pale cytoplasm with inter-follicular infiltration around the residual reactive or colonized germinal centers (Fig. 1C).

Immunohistochemical study was performed using the labeled streptavidin-biotin peroxidase method (LSAB kit, DakoCytomation, Carpinteria, CA, USA), and an antigenretrieval technique was applied as needed for each specific antibody. The antibodies used were CD3, CD20 (clone L26), CD21 (1F8), bcl-2 (124), bcl-6 (PG-B6p), Ki-67 (MIB-1), IgD, IgM, MUM1 (MUM1p (DakoCytomation), CD43 (MT1) (BioGenex, San Ramon, CA, USA), CD5 (4C7), CD10 (56C6), CD23 (1B12), CD27 (137B4) (Novocastra, Newcastle upon Tyne, UK), cyclin D1 (SP4; LAB Vision Co., Fremont, CA, USA), p53 (DO-7) (Serotec Inc., Raleigh, $\mathrm{NC}$, USA) and bcl-10 (in-house) [9]. Staining with antiCD21 highlighted the follicular dendritic meshworks in the altered or colonized germinal centers, which were widely spaced due to inter-follicular infiltrate. The tumor cells expressed CD20, bcl-2, and IgM, but not CD3, CD5, CD10, CD23, CD27, CD43, bcl-6, IgD, cyclin D1, p53, or MUM1. Staining for bcl-10 showed weak to moderate cytoplasmic 

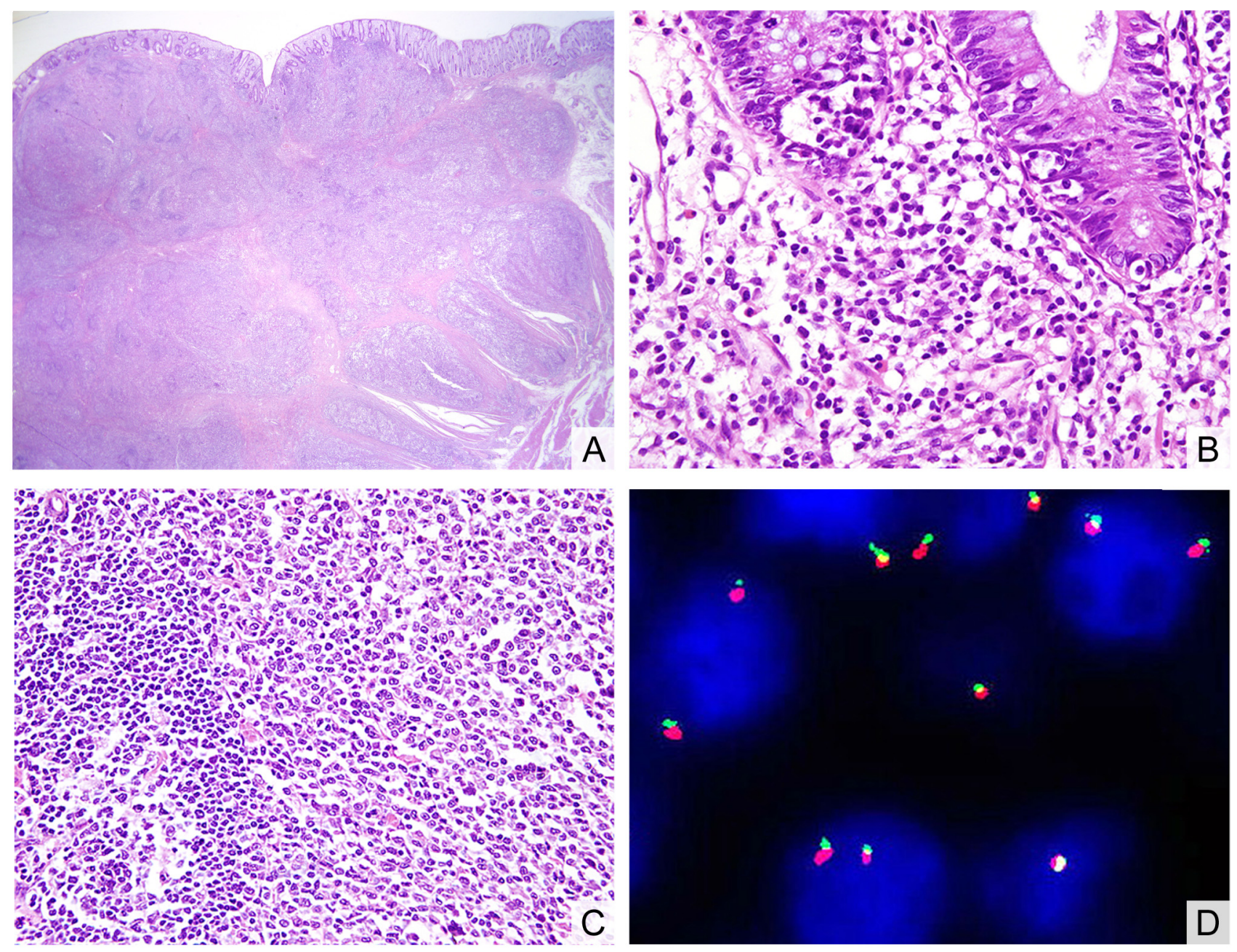

Fig. (1). Primary rectal mucosa-associated lymphoid tissue lymphoma. (A) Low-power view shows a polypoid lymphoid infiltrate with intact surface mucosa and extension into muscularis propria at the right lower corner. (B) Focal lymphoepithelial lesion. (C) High-power view of the atypical lymphocytes with slightly irregular nuclear contours on the right side with residual germinal center on the left. (D) Fluorescent in situ hybridization using MALT1 dual color, break apart rearrangement probe. In tumor cells with MALT1 rearrangment, there will be one fusion, one orange, and one green signal pattern indicative of a rearrangement of one copy of the MALT1 gene region while two fusion signal pattern indicates negative results for translocation. In our case, the image shows two fusion signal pattern indicating negative results for MALT1 rearrangement.

staining without nuclear signals. The proliferation fraction as determined by Ki-67 immunostaining was very low.

Locus-specific interphase FISH was performed on paraffin sections. Briefly, de-paraffinized sections were pretreated by pressure-cooking for 3 minutes in EDTA (ethylenediaminetetraacetic acid) buffer $(1 \mathrm{mM}, \mathrm{pH} 8.0)$ and subsequent incubation in pepsin solution for 25 minutes at $37^{\circ} \mathrm{C}$ to increase DNA accessibility. Sections were then dehydrated through ethanol and air-dried. The appropriate probe mix $(1.0 \mu 1)$ was applied to the tissue section and covered with a round $10 \mathrm{~mm}$ cover slip. Both probe and target DNA were simultaneously denatured at $80^{\circ} \mathrm{C}$ for 25 minutes and incubated up to 2 days at $45^{\circ} \mathrm{C}$. Post-hybridization washes were performed according to the "rapid-wash protocol" provided by Vysis, Downers Grove, IL. Sections were counterstained with 4,6-diamidino-2-phenylindole (DAPI) and mounted in Vectashield antifade solution (Vector Laboratories, Burlingame, CA, USA). Image acquisition and processing was per- formed as previously described [10]. Six dual color, break apart rearrangement probes directed at $I G H, M A L T 1, B C L 2$, BCL6, CCND1 (Vysis/Abbott Laboratories Ltd, UK), and FOXP1 (in-house) [11] genes and three centromeric enumeration probes (CEP) directed at chromosomes 3, 12 and 18 (Vysis/Abbott Laboratories Ltd.) were used. The tumor cells showed negative results for rearrangements involving IGH, MALT1 (Fig. 1D), BCL2, BCL6, and CCND1 genes. Repeated study using probes for $F O X P 1$ rearrangement was unsuccessful. All three FISH assays using CEP 3, 12, and 18 probes showed two dots, indicating absence of trisomies 3 , 12 , or 18 .

\section{DISCUSSION}

The differential diagnoses of this case include follicular lymphoma (FL) and mantle cell lymphoma (MCL). In addition to morphologic features, immunophenotype is helpful in excluding these two diagnoses by the negative expression of 
CD10 and bcl-6 for FL and CD5, CD43, and cyclin D1 for MCL.

MALT lymphoma is associated with recurrent chromosomal translocations $\mathrm{t}(11 ; 18)(\mathrm{q} 21 ; \mathrm{q} 21), \mathrm{t}(14 ; 18)(\mathrm{q} 32 ; \mathrm{q} 21)$, $\mathrm{t}(1 ; 14)(\mathrm{p} 22 ; \mathrm{q} 32)$, and $\mathrm{t}(3 ; 14)(\mathrm{p} 14.1 ; \mathrm{q} 32)$ involving $A P I 2$ (11q21), IGH (14q32), MALT1 (18q21), BCL10(1p22), and FOXP1(3p14) genes $[12,13]$. Rectal MALT lymphoma is very rare and usually appears as single case reports in the literature. In the earlier studies, two rectal MALT lymphomas with conventional cytogenetic studies were reported. Both tumors carried $\mathrm{t}(11 ; 18)$, one as the sole chromosomal aberration and the other with an additional aberration of trisomy $3[6,7]$. Using multiplex reverse transcription polymerase chain reaction (RT-PCR) method, Sakugawa et al. identified API2-MLT1 fusion genes in 7/47 (15\%) colorectal MALT lymphomas including 3/27 (11\%) primary rectal tumors [8]. As compared to the API2-MLT1-negative cases, API2-MLT1-positive colorectal MALT lymphomas were larger in size, with male predominance and advanced clinical stages. In a most recent case report with literature review, Kabayashi et al. reported API2-MLT1 fusion gene by RTPCR in a young adult with primary rectal MALT lymphoma successfully treated with radiotherapy [5]. In that review of a total of 34 cases, the median age is 62 years with equal sex ratio and a predominance of polypoid tumors. Ulcerative lesion is far less common, while multiple mucosal lesions with simple reddish discoloration as reported by Lee et al. are extraordinary [14].

Using extensive FISH probes, we intended to look for other possible chromosomal translocations specific for MALT lymphoma in our case since so far no cytogenetic/molecular genetic studies other than $\mathrm{t}(11 ; 18) / A P I 2-$ $M L T 1$ have been reported. We found that our case was negative for rearrangements involving IGH, MALT1, BCL2, $B C L 6$, and CCND1 genes while the study for FOXP1 gene rearrangement was unsuccessful. The failure to get satisfactory FOXP1 signals may be due to sub-optimal quality of this in-house probe as compared to the commercial probes, although it had been successfully applied to paraffin sections previously $[10,15]$.

In summary, our patient had a solitary and polypoid rectal MALT lymphoma presenting with bowel habit change. He was cured with polypectomy, radiotherapy and low dose chemotherapy. No specific chromosomal translocation or trisomy commonly associated MALT lymphoma was identified by extensive FISH study. More reports of rectal MALT lymphomas with extensive searches for chromosomal aberrations and with long-term follow-up data such as reported here will help to uncover the pathogenesis, best treatment strategy and clinical course of such tumors.

\section{ACKNOWLEDGEMENT}

This work was supported by research grant CMFHR9635 from Chi-Mei Medical Center.

\section{REFERENCES}

[1] Isaacson PG. Gastrointestinal lymphomas of T- and B-cell types. Mod Pathol 1999; 12: 151-58.

[2] Domizio P, Owen RA, Shepherd NA, Talbot IC, Norton AJ. Primary lymphoma of the small intestine. A clinicopathological study of 119 cases. Am J Surg Pathol 1993; 17: 429-42.

[3] Radaszkiewicz T, Dragosics B, Bauer P. Gastrointestinal malignant lymphomas of the mucosa-associated lymphoid tissue: factors relevant to prognosis. Gastroenterology 1992; 102: 1628-38.

[4] Ahlawat S, Kanber Y, Charabaty-Pishvaian A, et al. Primary mucosa-associated lymphoid tissue (MALT) lymphoma occurring in the rectum: a case report and review of the literature. South Med J 2006; 99: 1378-84.

[5] Kobayashi T, Takahashi N, Hagiwara Y, et al. Successful radiotherapy in a patient with primary rectal mucosa-associated lymphoid tissue lymphoma without the API2-MALT1 fusion gene: a case report and review of the literature. Leuk Res 2008; 32: 173-75.

[6] Hosaka S, Akamatsu T, Nakamura S, et al. Mucosa-associated lymphoid tissue (MALT) lymphoma of the rectum with chromosomal translocation of the $\mathrm{t}(11 ; 18)(\mathrm{q} 21 ; \mathrm{q} 21)$ and an additional aberration of trisomy 3. Am J Gastroenterol 1999; 94: 1951-54.

[7] Yatabe Y, Nakamura S, Nakamura T, et al. Multiple polypoid lesions of primary mucosa-associated lymphoid-tissue lymphoma of colon. Histopathology 1998; 32: 116-25.

[8] Sakugawa ST, Yoshino T, Nakamura S, et al. API2-MALT1 fusion gene in colorectal lymphoma. Mod Pathol 2003; 16: 1232-41.

[9] Ye H, Dogan A, Karran L, et al. BCL10 expression in normal and neoplastic lymphoid tissue. Nuclear localization in MALT lymphoma. Am J Pathol 2000; 157: 1147-54.

[10] Martin-Subero JI, Chudoba I, Harder L, et al. Multicolor-FICTION: expanding the possibilities of combined morphologic, immunophenotypic, and genetic single cell analyses. Am J Pathol 2002; 161: 41320 .

[11] Haralabieva E, Adam P, Ventura R, et al. Genetic rearrangement of FOXP1 is predominantly in a subset of diffuse large B-cell lymphomas with extranodal presentation. Leukemia 2006; 20: 13001303.

[12] Isaacson PG, Du MQ. MALT lymphoma: from morphology to molecules. Nat Rev Cancer 2004; 4: 644-53.

[13] Streubel B, Vinatzer U, Lamprecht A, Raderer M, Chott A. $\mathrm{T}(3 ; 14)(\mathrm{p} 14.1 ; \mathrm{q} 32)$ involving IGH and FOXP1 is a novel recurrent chromosomal aberration in MALT lymphoma. Leukemia 2005; 19 : 652-58.

[14] Lee YG, Lee S, Han SW, Lee JS. A case of multiple mucosaassociated lymphoid tissue (MALT) lymphoma of the colon identified as simple mucosal discoloration. J Korean Med Sci 2005; 20: $325-28$.

[15] Nakamura S, Ye H, Bacon CM, et al. Translocations involving the immunoglobulin heavy chain gene locus predict better survival in gastric diffuse large B-cell lymphoma. Clin Cancer Res 2008 in press. 